\title{
Novel trends in optical non-destructive testing methods
}

P. Huke
huke@bias.de

\section{R. Klattenhoff \\ C. von Kopylow \\ R. B. Bergmann}

\author{
BIAS - Bremer Institut für angewandte Strahltechnik GmbH, Klagenfurter Straße 2, 28359 Bremen, \\ Germany \\ BIAS - Bremer Institut für angewandte Strahltechnik GmbH, Klagenfurter Straße 2, 28359 Bremen, \\ Germany \\ BIAS - Bremer Institut für angewandte Strahltechnik GmbH, Klagenfurter Straße 2, 28359 Bremen, \\ Germany \\ BIAS - Bremer Institut für angewandte Strahltechnik GmbH, Klagenfurter Straße 2, 28359 Bremen, \\ Germany
}

Non-destructive testing (NDT) describes a wide range of methods for measuring and comparing physical quantities against a nominal condition. In this paper we describe and compare different optical NdT (ONDT)-methods with respect to their characteristics and capability for different measurement tasks. ONDT may be specified in two categories, passive and active. The NDT principles of the first category just use a measurement method like view inspection, elipsometry or reflectometry to detect defects which are easily accessible. The principles of the second category use an excitation force, such as heat or mechanical vibration introduced by transducers to detect hidden defects. This category can be specified into two subcategories. The first subcategory "time-/depth-resolved" includes measurement methods delivering detailed information of the geometric features of a hidden defect. Therefore the excitation of the material and the detection of the reaction have to provide a time step which enables depth-solved measurements. Phase-resolved thermography and laser ultrasound are examples for this category. The second subcategory "Integrating" includes measurement technique coupled with an excitation that enables detection of defects but not evaluation of their geometric features. Examples for these measurement techniques are shearography, reflectometry, vibrometry and thermography coupled with excitation method like simple heating or loading with a constant force. We demonstrate experimental results obtained using methods developed in our institute and highlight directions of further development. [DOI: http://dx.doi.org/10.2971/jeos.2013.13043]

Keywords: Shearography, reflectometry, laser ultrasound, optical NDT

\section{INTRODUCTION}

Quality control includes a wide range of properties like shape, texture, material parameters, purity as well as parameters like functionality and usability. Most of the properties can and should be evaluated non-destructively either using implemented systems (structural health monitoring) or " offline" methods for testing [1]. Even though it is difficult to find a thorough definition, non-destructive testing (NDT) describes a group of methods generally connected to the measurement of one or more object, material or system properties and in a second step assessment against a nominal condition. From this assessment a statement can be derived if the observed property lies in a predefined tolerance range, which is the principle demand coming from the industry. Thus, industry tends to use the most simple technique available, which delivers the go or no-go decision based on a comparison. Unfortunately, a comparison is most often only possible when a full measurement of the property in question took place and the results were interpreted, compared to a second state and once again interpreted. This complicates the development of a measurement technique since the developer has always to forecast where the technique maybe used for. One can easily go one step further down the line and find the same obstacle between the development of the measurement technique and basic principles. And in these gaps trends tend to start, mostly motivated by the industry. This motivation comes partly from unsolved problems in well established production technologies but more often from new trends in engineering sciences. A few of these predefined application areas can be identified like fibre-reinforced plastics as new materials with new processing technologies and a range of new defects to be detected. Other trends come from biomaterials and micro technologies introducing new challenges to the NDT. For the application in most of these fields it is convenient to use a contactless and remote technique like optical NDT (ONDT) [2]. Formerly, ONDT was connected to optical metrology with emphasis on NDT and not as an own field in which engineering sciences and optical physics merges and blends with materials sciences in a new complex way. This accounts for the interaction of the measuring " force" , e.g. light, with the material as well as for the use of external loads to stimulate a detectable signal which is specific for this method and is connected to a defect. In the past years ONDT sees growing influence, since the technologies under development become feasible and the results reliable enough for a go- or nogo-decision. This is mainly based 
on the development of the used tools: laser, cameras and faster computers enabling handling of the large volumes of data encrypted in an optical measurement [3]. In the following we show a categorization of ONDT which was conceived along the way a customer would choose its technology with respect to the defect. Therefore we give a short introduction into detectable defects in the first place.

\section{Defects detectable with ONDT}

There exists a wide range of defects as a category of errors which may be classified by criterions related to the whole process from defect formation during production [4] or utilization of the product until the relevance to the customer. Defects are characteristic values which do not fulfill their requirement and maybe regarded as discontinuities. A classification and study on the origins of discontinuities in (compound) metals can be found in [1]. As can be seen the principle meaning of the word defect always relates to the material, the process or the object in question. The defects origin can be divided into operationally induced and production induced discontinuities [1]. The former induce a range of defects caused by fatigue, creep, brittle fractioning, humidity and wear and must be detected during maintenance. The production induced defects must be detected during quality control in between or at the end of the value-added chain. Object defects may be defined as " global" deviations from the requirement, e.g. false material used for the fabrication, geometric deviations from the desired shape or surface characteristics not in the tolerances (roughness, color, light reflection behavior). Consequently, material defects can be defined as "local" deviations from the requirement and are entirely connected to the material itself. When regarding to conventional (metallic) materials, a simple classification can be used as in [5]. The classification criterion is the depth in which the defect is present:

- Surface defects: local deviation of color, shape (scratches, grooves, dents, bumps) or material (impurities, residual stresses)

- Subsurface defects: cracks, (open) pores, cavities

- Deep-volume defects: cracks, pores or cavities.

This criterion is suitable with respect to the accessibility of the defects. In general those defects may be detected using well established methods and their detectability depend on size, orientation, depth, in the defect included material and material behavior. For material bonds like soldering and welding, defects are categorized within the DIN EN ISO 6520-I and DIN EN ISO 5617. Most of these defects are hidden so that commonly bonds are tested using conventional ultrasound or $\mathrm{X}-\mathrm{CT}$. Laser-generated ultrasound might be a solution to detect some of these defects [42]. Another spectrum of defects comes with new compound materials, especially fibre reinforced plastics (FRP). Their compositions are designed to have advantageous characteristics like high Young's modulus combined with low weight. As a consequence to their multilayered structure they show high anisotropy, which complicates NDT. Defects specific to these materials are holes in the fabric, pores, delaminations, contamination of the surface effectively weakening the material and residual stresses [6-8]. Those defects can be found with conventional ultrasound when using high testing frequencies. However the structure complicates the residual signal. There once again laser generated ultrasound might be convenient as well as pulsed thermography or shearography for larger defects [9-11]. Along with the new materials bonding technologies were adapted like adhesive bonding. The most critical defect in adhesive bonding is the "kissing bond". The residual strength of this contact is marginal. However, up to now only laser induced shock waves seems to be able to test this bonding [12,13]. As a consequence, optically induced wave generation may be identified as a new trend as it provides solutions to new problems. In the field of microtechnology there are more challenges to be found as a consequence to the sheer size of the objects in question and the even smaller dimensions of the defects. The bonding between wafers once again may be tested using laser induced shock waves [14], while small cracks in the lattice structure can be found by laser generated shock waves [36]. Minute spots on the surface and below tend to develop during production. The former may be detected using techniques like X-CT, atomic force microscopy or scanning electron microscopy [15] all cost and time intensive methods. The surface and subsurface defects can be detected far more efficient with shearography $[16,17]$, which is an active optical nondestructive testing.

\section{Optical non-destructive testing (ONDT)}

The major advantage of ONDT is to measure contactless and remote, despite the fact that some quantities (e.g. reflectivity, color) can only be measured optically. Measuring properties from a distance ensures that the state of the object does not influence the measurement system and enables capturing of properties of hot tubes [18] and in-process ONDT. The measurement process itself is always influenced by the object and material properties, e.g. a laser-excited Lamb wave propagating on a Carbon fibre reinforced plastic (CFRP)-part experiences different damping and velocity in different direction $[19,20]$. So, anisotropy of the material as well as temperature, stresses or even grain sizes of metals [21] influence e.g. the propagation of an ultrasound wave, giving rise to calibration procedures, simulations and test standards in industry. Generally the appropriate method for ONDT is chosen with respect to the property in question and to the optical appearance of the probe [26]. If the property does not fulfill the requested tolerances, e.g. too many scratches, too deep grooves on the surface or other defects, the object is declared defective. The respective ONDT-method must be able to measure this property in a resolution sufficient for a qualifying decision. A convenient but by no means complete categorization from the viewpoint of the developer or distributor is shown in Figure 1.

On the first level the different NdT methods are discerned according to their physical " approach" . On the second level, the ONDT are differentiated as being either passive or active. "Passive" methods can be any optical measurement technique 


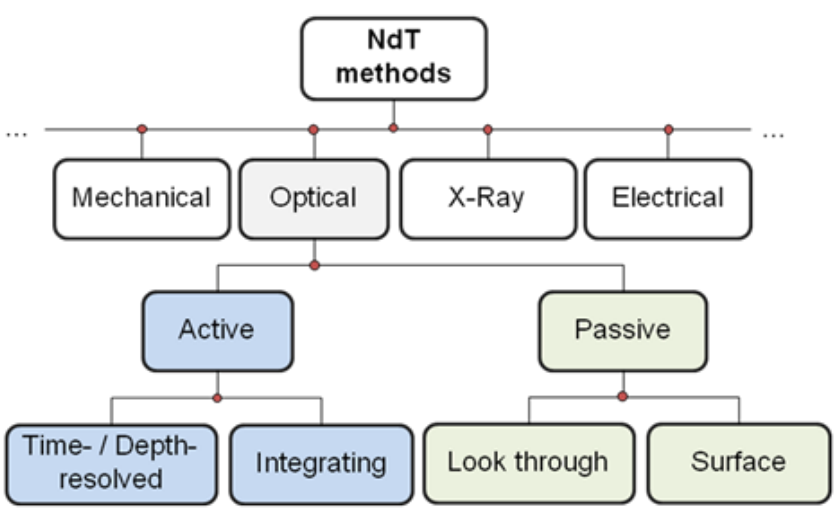

FIG. 1 Categorization of NdT methods by technology. First level: Physical approach. Second level: Active excitation with an extra force or passive capturing of signals. Third level: In-depth resolution.

producing a signal related to the examined property like elipsometry, reflectometry, photogrammetry, interferometry, etc. Good overviews are given by [22, 23, 27]. They divide into the surface related methods, measuring surface properties like reflectivity, shape, texture, roughness, etc. and look through method, like the quality of control of (micro-)lenses with interferometry [24]. "Active" methods are combined methods (sometimes called nondestructive load testing). They use a passive optical measurement technique and combine it with a certain load in order to excite a signal related to the property. For hidden defects the defect detection can be divided into four steps:

\section{Measurement of the object (e.g. surface, shape)}

\section{Loading (thermal, mechanical, electrical)}

\section{Measurement(s) of the object under load or thereafter}

4. Retrieval of defect related surface deviation (e.g. deformation, vibration, thermal flux)

As can be seen these methods are related to material purity and structure and are mostly used to search for defects hidden inside the material. If the material would be transparent and the defect can be detected without further excitation a (passive) look through method would be applicable. Loading can be achieved by different forces: Thermal (from simple heating to pulsed-phase-resolved), vibrant (e.g. with a piezoelectric or electromagnetic transducer, ultrasound guided through air), chemical (producing a color change on the surface), electrical (indirect heating using eddy currents) and so on. The choice of the appropriate loading depends on material properties as well as the property in question as can be seen from [25]. On the third level, active methods are divided into time/depth-resolved and integrating methods effectively differentiated by the time-step resolution of the system (load + measurement technique). As a general rule the complexity and costs of methods in the third level rise from right to left. In the following section the differences of both active methodscategories is described.

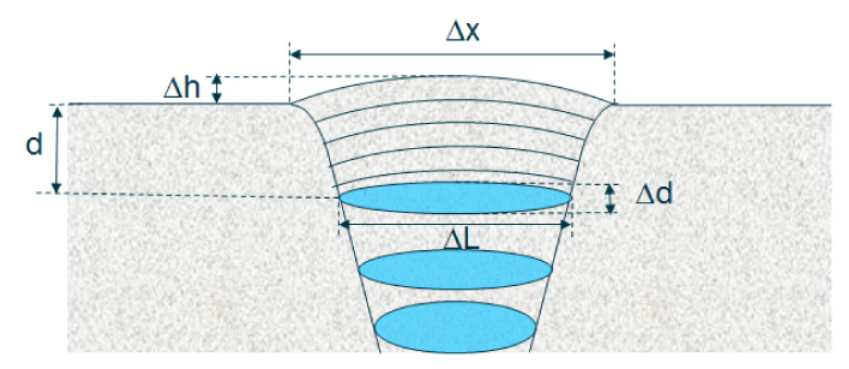

FIG. 2 2D-sketch of a full body deformation caused by heating. Defects of different height $\Delta d$ and length $\Delta L$ hidden in the depth d cause the same surface deviation $\Delta x$ and $\Delta h$ respectively.

\subsection{Integrating methods}

\subsubsection{General considerations}

In this section, we demonstrate the modelling results followed by some discussions. For a $100 \%$-quality control often it suffices to know if there is a defect or a weakness in the inner structure of a body or a compound. The object is discarded as soon as a defect is located regardless of its geometrical features. For integrating methods a typical combination would be thermal loading and a shape measurement technique like shearography, holography or reflectometry, all of them capable of measuring surface gradients in the range of a few nm [26]. While the object heats up, the surface deforms accordingly and a subsurface inclusion causes a local deviation which is slightly different from the surrounding. Even if the surface is measured several times at different temperatures it is in general not possible to determine the depth in which the defect is buried. Therefore the extraction of geometrical features pose an inverse problem [27] as can be seen from Figure 2. The size of the surface deviation $\Delta x$ is proportional to $\Delta L$ and inversely proportional to the depth $\mathrm{d}$ in which the defect is hidden. In the same way the height of the surface deviation $\Delta h$ is proportional to the defects height $\Delta d$ and once again inversely proportional to the depth $\mathrm{d}$. So these are two relations with three unknown variables which can be reduced to one equation with two unknown variables and two measurables. This simplified phenomenological description of the proportionalities neglects material related phenomena like anisotropy, which may help to solve the problem. For some cases, like the influence of the depth of cracks on Rayleigh waves, the problem can be solved (two-dimensionally) and the geometrical features can be reconstructed [28].

\subsubsection{Loads for integrating methods}

Two forces are typically exerted within the integrating methods: Thermal and mechanical loading. Thermal loading can be generated contactless and is therefore suitable for a wide range of objects and materials [26, 29,38]. For shape measurement different techniques can be applied, e.g. shearography (suited for rough and smooth surfaces), reflectometry (suited for reflective surfaces) [26] and holography (for small objects) [30]. Excitation and measurement of thermal sources is the basis for transient thermography which measures the cooling behavior of a body [31]. It is based on the influence of inhomogeneities or defects on the thermal flow caused by the heat- 


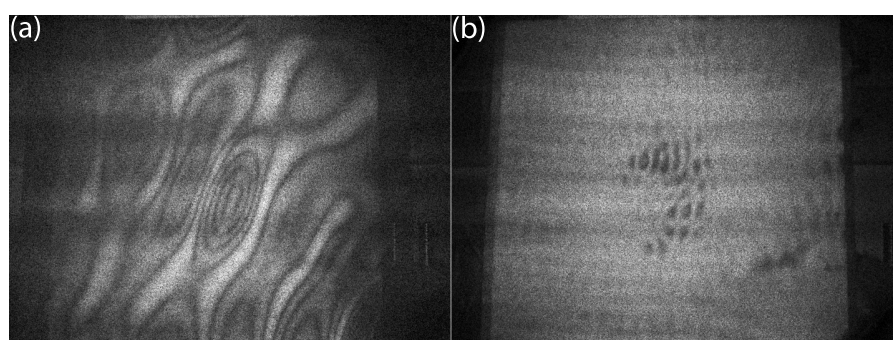

FIG. 3 Eigenfrequencies on a carbon-fibre reinforced (CFRP) plate (300 mm $\times 300 \mathrm{~mm} \times 2 \mathrm{~mm}$ ) measured with the shearography sensor shown in Figure 3. a) Excitation at $1.5 \mathrm{kHz}$, shear $\times 100$, the whole plate is vibrating. b) Excitation at $23.6 \mathrm{kHz}$, shear $\times 100$, material defects enclosed in the center of the plate resonate. Measured with shearography.

ing source. As the interface between the measurement object and the atmosphere is changing the flow dramatically, thermography cameras observe the flow on the surface of the object. By subsequent time stepping the alteration of the thermal flow can be used to get a qualitative statement on the depth in which a defect is hidden. However, the depth and the exact geometrical features cannot be derived exactly without further knowledge as stated in the previous section. Vibrational loading can be applied to nearly every solid object either by pulsed excitation or by excitation with continous waves. The latter one is commonly realized with an electromechanic or piezoelectric transducer often allowing for a range of adjustable frequencies [32]. Depending on the material or defect properties resonant frequencies can be excited showing distinctive vibrational states, see Figure 4.

In general, the defect detection with vibrational loading and a shape measurement method like shearography needs a trained worker or a sophisticated image processing software. In the past, different approaches to extract geometrical features of defects from distinctive vibrational pattern were developed [32-34]. Even though these approaches show good results for simple cases, reliable reconstruction in a volumetric body remains a challenge. Pulsed excitation of surface or full body waves is done two-dimensionally by either using a piezoelectric transducer or by a short pulsed laser. The detection can be done by holography [35], shearography [19], vibrometry or one-dimensionally by a pump-probe technique [36]. In BIAS a setup was developed for the observation of Lamb waves on carbon-fibre reinforced plastics (CFRP). These waves are influenced by the materials anisotropy, as can be seen in Figure 5, the pre-stressed state [20] and defects on and below the surface.

In the following sections shearography is used as an example for integrating methods.

\subsubsection{Shearography}

As with all active, integrating ONDT principle two images are taken, one without excitation and one is a certain load, exciting a surface deviation caused by the hidden defect. The superposition delivers the change of the surface gradient. A local defect below the surface alters the surface gradient. Figure 4
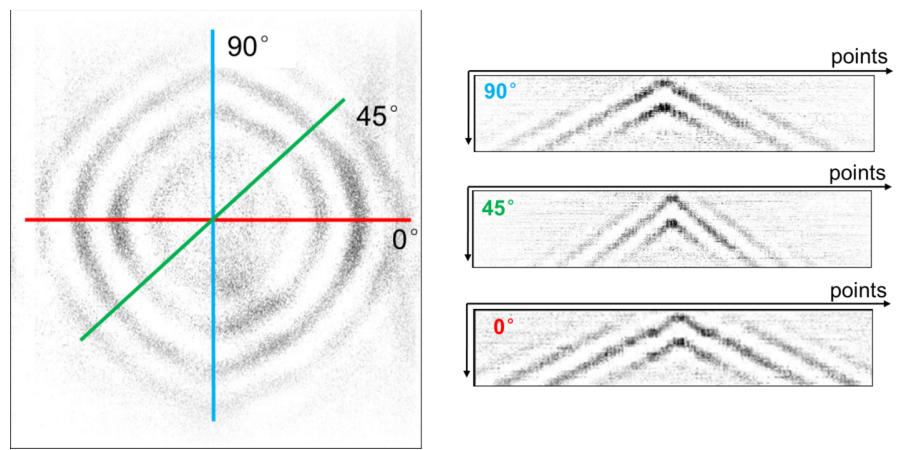

FIC. 4 Lamb waves on CFRP $0^{\circ}-90^{\circ}-0^{\circ}-90^{\circ}$ recorded with shearography. a) Snap shot of a propagating wave. b) Time-space (here by measurement points) plot show the anisotropy: the propagating wave in $45^{\circ}$-direction is much slower than in $0^{\circ}-$ and $90^{\circ}$-direction.

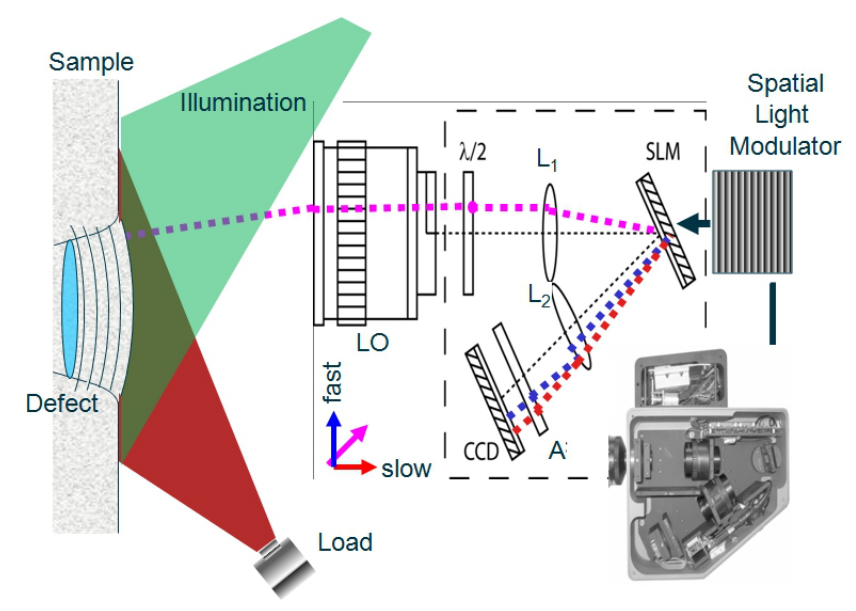

FIG. 5 Principle setup of the shearography sensor developed at BIAS [29]. For simplification a single beam coming from the (linear polarized) illumination is reflected into the sensor. With the objective lens (LO) in front a larger area can be inspected. The $\lambda / 2$-plate provides circular polarization. With the spatial light modulator the beam is separated into two orthogonally polarized beams, which illuminate different pixel on the CCD. The position of the spatial light modulator is in the Fourier plane of a 4 -setup.

shows the principle setup of the Shearography-sensor developed at BIAS [29].

The sample is illuminated by an expanded laser beam linearly polarized. After the objective lens the plane of polarization is aligned using the $\lambda / 2$-plate. The spatial light modulator in the Fourier plane of the lens L1 affects only the part of the beam with one plane of polarization while the other perpendicular plane is directly reflected. The two beams are then laterally shifted and illuminate different pixels on the CCD. The obtained image of the full field of view is sheared and the shear can be controlled by the spatial light modulator. Figure 3 shows the experimental setup consisting of a specimen provided by conservator Siegfried Herrmann, a simple heating device and the Shearography sensor [37]. The specimen was produced using the stucculustro-technique and damaged afterwards. On top of the damaged surface is a plaster layer hiding the damages. This specimen simulates a situation often experienced by conservators. In the left bottom of the figure the detected defects below the surface of the specimen are shown on the controlling PC. 


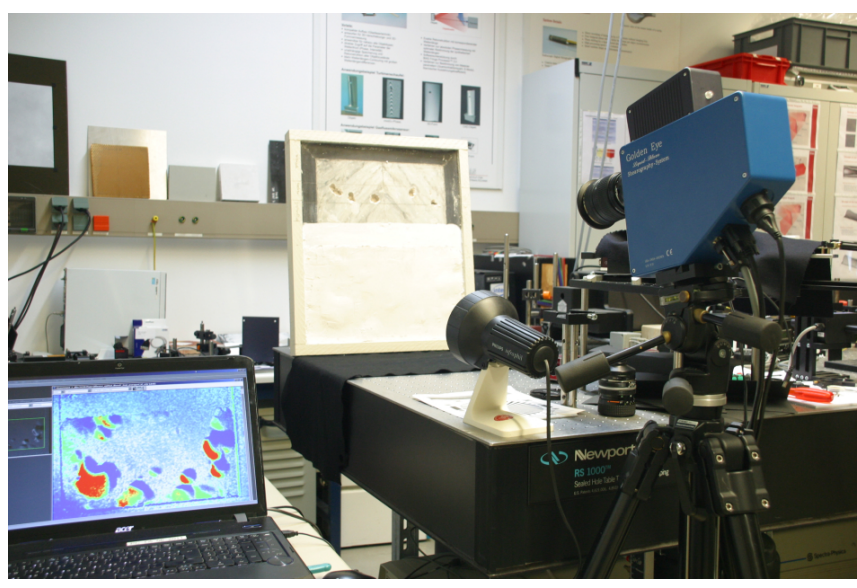

FIG. 6 Experimental setup consisting of a specimen provided by conservator Siegfried Herrmann, a simple heating device, and a shearography sensor based on the liquid blaze technology.

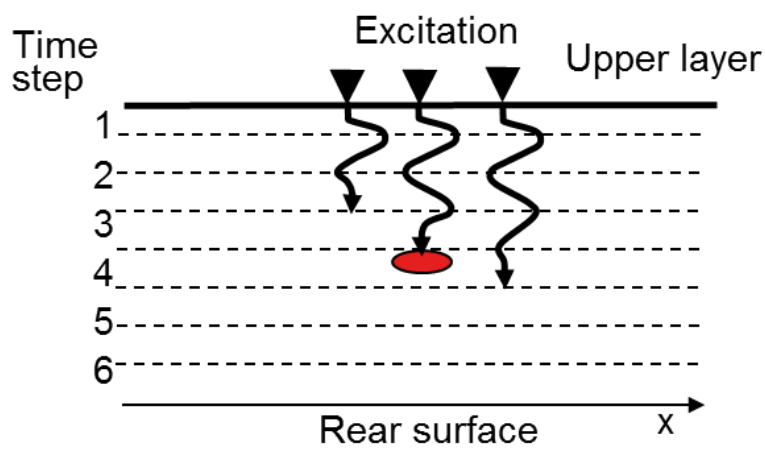

FIG. 7 Principle sketch of a time stamped excitation / detection. The depth can be resolved by the time steps. Heat or ultrasound waves travel through the measurement object until they reach a defect or the rear surface. The reflected signal can be measured phase-resolved.

\subsection{Time- / Depth-resolved Methods}

\subsubsection{General considerations}

A method is grouped into the time- / depth-resolved criteria when it includes short, discriminable time steps allowing for resolving defects in the depth direction of a body. The time stamp has to be generated during or by the loading and must be maintained during measurements, see Figure 6 .

A typical method for this class is time-gated or phase-resolved thermography with different excitation of thermoelastic waves like optical- (OLT), ultrasound- (ULT), induction-(ILT) lock-in thermography [38-41]. Another frequently used type of loading are elastic waves travelling through the object. The surface deviations can be detected using Doppler-vibrometry using an off-the-shelf vibrometer or by a designed FabryPerot cavity [42]. In order to get a sufficient resolution the measurable time steps have to be small enough and therefore the frequency of the ultrasound waves high enough. As already stated in [26] the resolution for defects depend in general on the wavelength of the ultrasound waves. Again as in the integrating methods, the ultrasound waves can be excited using a piezoelectric crystal or a short-pulsed high-power laser.

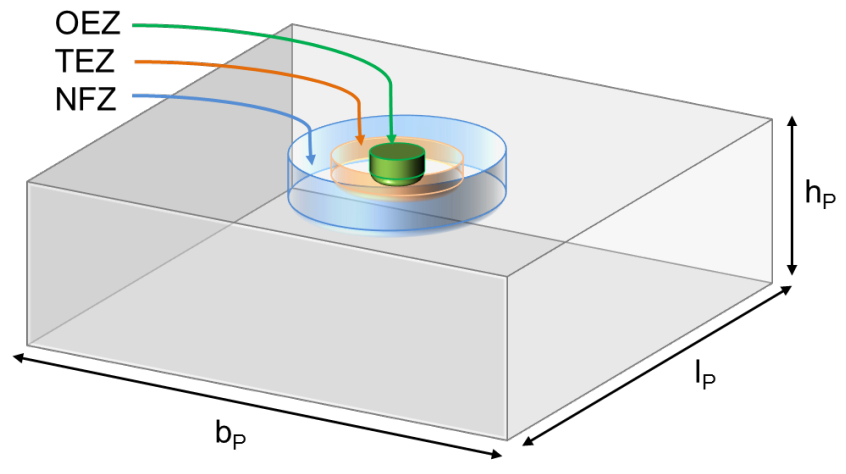

FIG. 8 Laser-material interaction. The incident laser pulse produces an optical penetration zone (OEZ) which in turn results in a thermoelastic zone (TEZ). The resulting ultrasound waves can be divided in a near-field zone (NFZ) and a far-field zone depending on the excited waves.

\subsubsection{Laser-generated ultrasound}

Laser-ultrasound is the excitation of ultrasound waves (the load) with a short-pulsed (pump-) laser and the detection of the resulting surface deviations with another (probe-) laser $[18,42,43]$. Since the ultrasound waves can be measured with high accuracy and a high time resolution this method delivers comparable and depending on the object under investigation more detailed results than conventional ultrasound [42]. For a pump-laser any short-pulsed high power laser with sufficient absorption in the material can be used. If no sacrificial layer is used, the absorbed energy of the pump-laser develops a thermoelastic stress-zone, see Figure 7.

The energy in this zone dissipates through thermal stresses as well as ultrasound waves in different directions depending on the laser and material parameter and the therefore excited frequencies [43-45]. The excitation is in general broadband which is convenient for the detection of larger defects or thickness measurements. As with conventionally excited ultrasound, there are also advantages of using narrow-band excitation as the detector can be tuned specifically to the excited frequencies and smaller defects especially in multilayered structures can be detected [46-48]. The surface deviations caused by the ultrasound waves modulate the frequency of a probe-laser. The detection-scheme behind the probe-laser can be a designed Fabry-Perot-cavity to filter the sidebands or a Vibrometer [18, 20,28].

\section{SUMMARY}

In this paper a classification of optical non-destructive testing (ONDT) methods is described from the viewpoint of a systems developer. As the development is oriented at the customers demands, the appropriate class for a specific task can be derived on the first level by physical approach, on the second level by the property in question (hidden defects generally need an active ONDT) and on the third level by the resolution needed (defect detection can be done by integrating methods, defect geometry can be measured with time-/depthresolved methods). For different classes examples were presented in order to illustrate the characteristics of the specific class. 


\section{ACKN OWLEDGEMENTS}

The authors would like to thank S. Herrmann, C. Falldorf, C. von Kopylow, O. Focke and M. Kalms at BIAS for their valuable contributions. Long term collaborations with industrial partners, mainly VEW Corp. (Vereinigte Elektronikwerkstätten), Satisloh Corp., and Airbus Corp. have substantially stimulated this work. Financial support of the following organizations is gratefully acknowledged: Part of this work was performed within the EU projects EVK4-CT-2002-00096 and GRD1-2000-25309, the German BMWi (Federal Ministry of Economics and Technology) projects KF0086401WM4 and 20W9904 and the DFG (German Research Foundation) projects Ju142/54-1 and Ju142/60-1.

\section{References}

[1] C. Hellier, Handbook of nondestructive Evaluation (McGraw-Hill, New York, 2012).

[2] A. Blouin, S. Kruger, D. Levesque, and J. Monchalin, "Applications of Laser-Ultrasonics to the Automotive Industry," in Proceedings to 17th World Conference on Non Destructive Testing, (WCNDT, Shanghai, 2008).

[3] P. Rastogi, and D. Inaudi, Trends in optical Non-destructive testing and inspection (Elsevier, Amsterdam, 2000).

[4] N. Rütthard, Rechnerunterstützter Erfahrungsrückfluß in der Prozesskette der Blechteilefertigung und -verarbeitung (Universität Hannover, Hannover, 2001).

[5] R. Bergmann, and P. Huke, "Advanced Methods for optical Nondestructive Testing," in Optical Imaging and Metrology: Advanced Technologies, 393-412 (Wiley, New Jersey, 2012).

[6] P. Parlevliet, H.Bersee, and A. Beukers, "Residual stresses in thermoplastic composites - A study of the literature - Part II: Experimental techniques," Compos. Part A-Appl. S. 38, 651-665 (2007).

[7] J. Svanberg, and J. Holmberg, "An experimental investigation on mechanisms for manufactuirng induced shape distortions in homogeneous and balanced laminates," Compos. Part A-Appl. S. 32, 827-838 (2001).

[8] C. Ramadas, K. Balasubramaniam, M. Joshi, and C. Krishnamurthy, "Interaction of guided Lamb waves with an asymmetrically located delamination in a laminated composite plate," Smart Mater. Struct. 19 (2010).

[9] S. John , "Non-Destructive Testing of Fibre-Reinforced Plastics Composites," Elsevier Applied Science 2, 57-68 (1987)

[10] F. Santos, M. Vaz, and J. Monteiro, "A new set-up for pulsed digital shearography applied to defect detection in composite structures," Opt. Laser Eng. 42, 131-140 (2004).

[11] M. Seale, and B. Smith, "Lamb wave propagation in thermally damaged composites," Rev. Prog. Q. 15A, 261-266 (1996).

[12] R. H. Bossi, K. R. Housen, W. B. Shepherd, and M. E. Voss, US Patent 6,848,321 B2 (2005)

[13] R. Bossi, K. Housen, and W. Shepherd, "Using Shock Loads to Measure Bonded Joint Strength," Mater. Eval. 60, 1333-1338, (2002)

[14] G. Youssef, C. Moulet, M. Goorsky, and V. Cupta, "Inter-wafer bonding strength characterization by laser-induced shock waves" J. Appl. Phys. 111, 094902 (2012)
[16] G. Udupa, W. Jun, and K. Bryan, "A combined fiber optic digital shearography and holography system for defect inspection in Siwafers," Proc. SPIE 5852, (2005)

[17] G. Udupa, B. Ngoi, H. Goh, and M. Yusoff, "Defect detection in unpolished Si wafers by digital shearography," Meas. Sci. Technol. $15,35-43$ (2004).

[18] J. Monchalin, C. Neron, J. Bussiere, P. Bouchard, C. Padioleau, R. Heon, M. Choquet, J. Aussel, G. Durou, and J. Nilson, "Laserultrasonics: from the laboratory to the shop floor," Adv. Perform. Mater. 5, 7-23 (1998).

[19] O. Focke, A. Hildebrand, C. Kopylow, and M.Calomfirescu, "Inspection of lamb waves in carbon fiber composites using shearographic interferometry," Proc. SPIE 6934, (2008)

[20] P. Huke, O. Focke, C. Falldorf, C. von Kopylow, and R. Bergmann, "Contactless Defect Detection using Optical Methods for Non Destructive Testing," in Proceedings of the 2nd Symposium on NdT in Aerospace (DGZfP, Hamburg, 2011).

[21] S. Sundin, D. Artymowicz, "Direct measurement of grain size in low-carbon steels using the laser ultrasonic technique," Metall. Mater. Trans. A 33A, 687-691 (2002).

[22] E. Savio, L. De Chiffre, and R. Schmitt, "Metrology of freeform shaped parts," CIRP Ann.-Manuf. Techn. 56, 810-835 (2007).

[23] F. Chen, G. Brown, and M. Song, "Overview of three-dimensional shape measurement using optical methods," Opt. Eng. 39, 10-22 (2000).

[24] F. Charrière, J. Kühn, T. Colomb, F. Monfort, E. Cuche, Y. Emery, K. Weible, P. Marquet, and C. Depeursinge, "Characterization of microlenses by digital holographic microscopy," Appl. Optics 45 , 829-835 (2006).

[25] IAEA, Handbook on non-destructive testing of concrete structures (CRC Press, Boca Raton, 2002).

[26] R. Bergmann, T. Bothe, C. Falldorf, P. Huke, M. Kalms, and C. von Kopylow, "Optical metrology and optical non-destructive testing from the perspective of objectcharacteristics," Proc. SPIE 7791, 1-15 (2010)

[27] W. Osten, "Digital Image Processing for Optical Metrology" in Springer Handbook of Experimental Solid Mechanics, Ed. Sharpe, 481-563 (Springer, Berlin, 2008).

[28] A. Moura, A. Lomonosov, and P. Hess, "Depth evaluation of surface-breaking cracks using laser-generated transmitted Rayleigh waves," J. Appl. Phys. 103, 084911 (2008).

[29] C. Falldorf, S. Osten, C. Kopylow, and W. Jüptner, "Shearing interferometer based on the birefringent properties of a spatial light modulator," Opt. Lett. 34, 2727-2729 (2009).

[30] B. Kemper, S. Kosmeier, P. Langehanenberg, S. Przibilla, C. Remmersmann, S. Stürwald, and G. von Bally, "Application of 3D tracking, LED illumination and multiwavelength techniques for quantitative cell analysis in digital holographic microscopy," Proc. SPIE 7184, 71840R (2009)

[31] P. Maldaque, Nondestructive evaluation of Materials by Infrared Thermography (Springer Verlag, Berlin, 1995).

[32] W. Osten, W. Jüptner, and U. Mieth, "Knowledge based evaluation of fringe patterns for automatic fault detection," SPIE Interferometry, 256-268 (1993).

[33] W. Osten, F. Elandalousi, and W. Jüptner, "Recognition by synthesis - a new approach for the recognition of material faults in HNDE," Proc. SPIE 2861, 220-224 (1996).

[15] K. Boving, NDE Handbook (Butterworths, London, 2001). 
[34] C. Furlong, and J. Pryputniewicz, "Hybrid, experimental and computational, investigation of mechanical components," Proc. SPIE 2861, 13-24 (1996).

[35] K. Telschow, V. Deason, R. Schley, and S. Watson, "Imaging of Lamb Waves in Plates for Quantitative Determination of Anisotropy Using Photorefractive Dynamic Holography," Rev. Prog. Q. 18 (1999).

[36] P. Hess, and A. Lomonosov, "Solitary surface acoustic waves and bulk solitons in nanosecond and picoseconds laser ultrasonics," Ultrasonics 50, 167-171 (2010).

[37] P. Huke, S. Herrmann, C. Falldorf, and R. Klattenhoff, "Hilfreiche Blicke unter die Oberfläche," Restauro 8, 28-32 (2012).

[38] P. Maldaque, Nondestructive evaluation of Materials by Infrared Thermography (Springer Verlag, Berlin, 1995).

[39] A. Dillenz, "Ultraschall Burst-Phasen-Thermografie," MP Material Testing 43, 1-2 (2001).

[40] C. Zöcke, Quantitative analysis of defects in composite material by means of optical lockin thermography (Universität des Saarlandes and Universitä Paul-Verlaine Mertz, Saarbrücken, 2009).

[41] G. Riegert, Induktions-Lockin-Thermografie ein neues Verfahren zur zerstörungsfreien Prüfung (Institut für Kunststofftechnik der Universität Stuttgart, Stuttgart, 2007).
[42] C. von Kopylow, 0. Focke, and M. Kalms, "Laser Ultrasound - A flexible Tool for the Inspection of complex CFK Components and Welded Seams," Proc. SPIE 6616, 66163J, (2007).

[43] C. Scruby, and L. Drain, Laser Ultrasonics:Techniques and Application (Inst. of Physics Pub., Bristol, 1990).

[44] P. Zhang, C. Ying, and J. Shen, "Directivity patterns of laser thermoelastically generated ultra-sound in metal with consideration of thermal conductivity," Ultrasonics 35, 233-240 (1997).

[45] M. Dubois, P. Lorraine, B. Venchiarutti, A. Bauco, and R. Filkins, "Optimization of temporal and optical penetration depth for lasergeneration of ultrasound in polymer-matrix composites," Rev. Prog. Q., 287-294 (2000).

[46] G. Shi, C. Chen, J. Lin, X. Xie, and X. Chen, "Narrowband Ultrasonic Detection with High Range Resolution: Separating Echoes via Compressed Sensing and Singular Value Decomposition," IEEE T. Ultrason. Ferr. 59, 10 (2012).

[47] T. Stepinski, and M. Jonsson, "Narrowband ultrasonic spectroscopy for NDE of layered structures," in Proceedings of 16th World Congress of NDT (ABENDE, BINDT, Munich, 2004).

[48] T. Stratoudaki, J. Hernandez, M. Clark, and M. Somekh, "Cheap optical transducers (CHOTs) for narrowband ultrasonic applications," Meas. Sci. Technol. 18, 843-851 (2007). 